\title{
松墨天牛对共生真菌和病原真菌的差异性免疫 响应
}

张伟 ${ }^{1,2 \dagger}$, 孟洁 ${ }^{1,2 \dagger}$, 宁静 ${ }^{1,4 \dagger}$, 秦培君 ${ }^{1,2}$, 周娇 ${ }^{1}$, 邹振 ${ }^{1,2}$, 王燕红 ${ }^{1}$, 江 红 ${ }^{1}, F^{2}$ aheem $\mathrm{Ahmad}^{1,3}$, 赵莉鹤 ${ }^{*}$, 孙江华 $1,2^{*}$

1. 中国科学院动物研究所, 农业虫害鼠害综合治理研究国家重点实验室, 北京 100101;

2. 中国科学院大学, 北京 100049 ;

3. Department of Biosciences, COMSATS Institute of Information Technology, Islamabad 45550, Pakistan;

4. 河北大学生命科学学院, 保定 071002

$\dagger$ 同等贡献

* 联系人, E-mail: sunjh@ioz.ac.cn; zhaoll@ioz.ac.cn

收稿日期: 2017-08-15; 接受日期: 2017-08-23; 网络版发表日期: 2017-09-05

中国科学院战略性先导科技专项(批准号:XDB11050000)、林业公益性行业科研专项(批准号: 201204501)、国家重点研发计划(批准号: 2016YFC1200604, 2016YFD0500300)、国家高技术研究发展计划(批准号: 2014AA020529)、国家自然科学基金(批准号: 31572272,31370650, 31402013, 31221091, 31672291, L1524009)、中国科学院前沿科学重点研究项目(批准号: QYZDB-SSW-SMC014)和中国科学院学科发展战 略研究(批准号: 2015-SM-C-02)资助

摘要在中国, 入侵生物松材线虫进行传播的主要媒介昆虫松墨天牛(Monochamus alternatus) 可与一种中国本 地蓝变真菌Sporothrix sp. 1建立体外共生关系. 然而在天牛和真菌共生互作过程中, 松墨天牛对蓝变菌S. sp. 1 的 免疫响应却未见报道. 因此, 本文通过比较体外共生真菌S. sp. 1 和昆虫病原真菌球孢白僵菌(Beauveria bassiana) 感染松墨天牛蛹所产生的免疫反应, 发现天牛对两种菌的免疫应答方式具有显著性差异. 将真菌注射到刚化蛹 的天牛体内, S. sp. 1 感染的天牛不会死亡, B. bassiana感染的天牛则全部死亡. 转录组测序结果显示, B. bassiana 感染 $48 \mathrm{~h}$ 后的松墨天牛的差异表达基因数量是S. sp. 1感染的 2 倍. 值得注意的是, 在体外共生真菌感染的松墨 天牛免疫系统中起主导作用的是Toll和IMD信号通路; 而在昆虫病原真菌感染松墨天牛后, 只有 Toll信号通路上 调表达. 此外, 体外共生真菌S. sp. 1在感染天牛48 h 后可通过下调天牛的Toll通路和 IMD通路来产生免疫逃避. 本研究提供了松墨天牛转录组全测序数据, 为后续松墨天牛和其相关真菌互作研究提供了基础.

关键词松墨天牛, 共生真菌, 球孢白僵菌, 转录组测序, 免疫信号通路

松墨天牛(Monochamus alternatus (Cerambycidae: Coleoptera) $)$ 是一种危害松树的蛙干害虫 ${ }^{[1,2]}$, 也是导 致松树枯萎病(PWD)的致病生物松材线虫(Bursaphelenchus xylophilus) 的主要传播媒介 ${ }^{[3]}$. 松墨天牛可与
一种中国本地体外共生蓝变菌Sporothrix sp. 1发生共 生关系, 从而使 $S$. sp. 1通过附着于松墨天牛体表来 进行传播 ${ }^{[4,5]}$. 不同于昆虫病原真菌, S. sp. 1不仅可与 松墨天牛共生, 还能促进天牛的发育、提升天牛的存

引用格式: 张伟, 孟洁, 宁静, 等. 松墨天牛对共生真菌和病原真菌的差异性免疫响应. 中国科学: 生命科学, 2017, 47: 988-996, doi: 10.1360/N052017-00162

英文版见： Zhang W, Meng J, Ning J, et al. Differential immune responses of Monochamus alternatus against symbiotic and entomopathogenic fungi. Sci China Life Sci, 2017, 60: 902-910, doi: 10.1007/s11427-017-9102-y 
活率 ${ }^{[5]}$. 在自然界中, 许多昆虫与ophiostomatoid蓝变菌 发生共生关系 ${ }^{[0-9]}$. 与昆虫病原真菌不同的是, 大多数 体外共生真菌不但不会杀死它的寄主, 反而对寄主的 生长发育是有利的 ${ }^{[10]}$. 就共生关系而言, 昆虫允许体 外共生真菌在其表面共生的分子机制还有待阐明.

昆虫与微生物的共生关系包括体内共生和体外 共生 ${ }^{[10]}$. 相较于体内共生, 体外共生真菌由于不需应 对昆虫体内产生的免疫防御, 且外部环境更适于其生 长, 故而更为常见. 例如, 一种以真菌为食的蚂蚁与其 体外共生真菌为互利共生关系: 蚂蚁以取食真菌为生, 真菌则以蚂蚁的体表为其生长、扩散和自我保护的基 质 ${ }^{[11]}$. 同样的, 红脂大小冨的伴生真菌Leptographium procerum 具有很强的生物降解活性, 可以降低松树对 虫菌共生体的抗性 ${ }^{[7,12]}$. 一般来说, 大部分对于体外共 生真菌的研究都集中于其共生关系的阐明, 很少有侧 重于昆虫对体外共生真菌免疫反应的研究.

为抵抗病原微生物感染, 昆虫进化出由体液免 疫和细胞免疫组成的先天性免疫系统. 感染后, 免 疫系统可启动针对病原体的细胞免疫或体液免疫. 细胞免疫由血细胞介导, 包括吞噬作用、包被作用 和结节作用 ${ }^{[13,14]}$; 体液免疫主要依靠合成大量抗菌肽 (antimicrobial peptides, AMPs)、血淋巴凝集反应和黑 化反应 ${ }^{[15 ~ 17]}$. 免疫信号通路在识别微生物表面的病原 相关模式分子(pathogen associated molecular patterns, PAMPs) 后被激活 ${ }^{[18]}$, 主要包括Toll信号通路 ${ }^{[19]}$ 、IMD 信号通路 ${ }^{[20]}$ 、JNK信号通路 ${ }^{[21]}$ 和JAK/STAT ${ }^{[22]}$ 信号通 路. Tol1信号通路和IMD信号通路是免疫应答的主要 调节因子 ${ }^{[23,24]}$. 抗菌肽基因主要通过两种不同的NF- $\mathrm{KB}$ 转录因子依赖型信号通路: Toll信号通路和IMD信号 通路的激活被诱导表达 ${ }^{[25]}$. Toll信号通路被真菌和 革兰氏阴性细菌激发, 由细胞外丝氨酸蛋白酶(serine protease, SP)级联介导. 感染后, Toll信号通路在血淋 巴中被激活, 导致Toll受体与其相应配体Spaetzle结合, 从而激活 $\mathrm{dMyD} 88$ 和激酶Pelle. 这会导致Cactus的降 解和NF-kB同源物Dif/Dorsal因子的释放. 而IMD途径 主要是由革兰氏阴性菌激发的, IMD途径的最终目标 是一种Rel/NF-KB 反式激活因子—Relish, 它被半胱 氨酸激酶Dredd和IKK切割, TAK是一种MAPKK激酶 (MAPKKK), 是一种受体相互作用蛋白, 在IKK的上游 和IMD的下游起作用 ${ }^{[23,26]}$. 通常, JNK和JAK/STAT通路 也可调节抗菌肽产生 ${ }^{[27,28]}$.
球孢白僵菌是一种广泛存在且研究较为透彻的 昆虫病原真菌 ${ }^{[29]}$. 作为农林害虫生物防治的经典昆虫 病原真菌, 球狍白僵菌对各种农林害虫具有较高的致 病活性, 对脊椎动物则基本上无毒性和传染性 ${ }^{[30]}$. 当 球狍白僵菌感染昆虫时, 会引起昆虫的细胞免疫和体 液免疫并激活寄主的Toll信号通路 ${ }^{[31,32]}$; 最终, 球狍白 僵菌会克服宿主的免疫反应并杀死宿主. 因此, 为了 解松墨天牛对体外共生真菌免疫耐受的潜在机制, 本 实验比较了松墨天牛蛹对 $S$. sp. 1和球狍白僵菌的免 疫响应. 本课题组还采用了无参考基因组的高通量 RNA-seq技术, 来探寻感染两种不同真菌的松墨天牛 的免疫反应的性质. 本研究旨在揭示感染外共生真菌 和昆虫病原真菌的松墨天牛蛹所产生的不同免疫反 应, 为进一步研究宿主和共生真菌之间的免疫相互作 用提供有价值的参考信息.

\section{1 材料与方法}

\section{1 天牛饲养和真菌培养}

实验室所用松墨天牛为采自浙江的种群, 在室内 饲养多代. 幼虫在无光照 $25^{\circ} \mathrm{C}$ 培养条件下以人工饲料 喂养, 使用刚化蛹天牛进行生物测定.

真菌在马铃薯葡萄糖琼脂培养基(potato dextrose agar, PDA)上以 $25^{\circ} \mathrm{C}$ 和 $80 \%$ 相对湿度条件培养 1 周. 本 实验所用的蓝变菌(CMW29982)是一种中国本地产真 菌, 标本保存于南非比勒陀利亚林业与农业生物技术 研究所(Forestry and Agricultural Biotechnology Institute, FABI). 球狍白僵菌的菌株号是ARSEF2860.

\section{2 真菌感染天牛存活率测定}

为使共培养试验条件更接近野外, 使用韧皮细粉 与MEA混合制成树皮粉培养基培养 S. sp. 1 和球孢白 僵菌. 将刚化蛹第一天的松墨天牛(10头一组)放置于 长有 $S$. sp. 1或球狍白僵菌的培养基上; 然后在 $25^{\circ} \mathrm{C}$ 无 光照条件下培养平板, 并观察天牛每天的生长状况, 记录感染真菌的死亡天牛数, 计算其死亡率. 本实验 重复3次, 使用 GraphPad软件进行数据分析.

\section{3 真菌注射天牛的生存分析以及 RNA样品制备}

在化蛹第 1 天, 将同一批天牛随机分为 3 组, 每组 10 个重复. 对照组在腹部第二体节处注射 $2 \mu \mathrm{L}$ 无菌 
PBST, 处理组注射 $2 \mu \mathrm{L}$ 稀释的真菌 (S. sp. 1/球孢白傈 菌)悬浮液 $\left(3 \times 10^{6}\right.$ 分生孢子 $\left./ \mu \mathrm{L}\right)$. 蛹培养于 $25^{\circ} \mathrm{C}$ 条件下, 分别培养 24,48 和 $72 \mathrm{~h}$, 并统计每组蛹的死亡率. 在体 式显微镜下解剖被 $S$. sp. 1 和球孢白僵菌感染 24 和 $48 \mathrm{~h}$ 的蛹的脂肪体组织用于RNA样本制备. 注射PBST的 对照组选取注射 $48 \mathrm{~h}$ 的蛹, 用同样的方法制备样品. 将 RNA组织样品置于 $1 \mathrm{~mL}$ TRizol Reagent (Invitrogen, 美 国)中按试剂说明书进行总RNA提取. 使用ND-1000 Spectrophotometer (NanoDrop Technologies, Inc., 美国) 进行RNA浓度测定. 每个样品为 3 头天牛脂肪组织的 混样.

\section{4 文库构建和Illumina测序}

用磁珠Dynabeads ${ }^{\circledR}$ 对总RNA进行纯化, 其原理为 大多数mRNA的poly(A) 尾与磁珠表面的oligo (dT)按 照碱基互补配对原则进行结合. 使用RNA-Seq Library Preparation Kit for Whole Transcriptome Discovery试剂 盒进行转录组文库构建 ${ }^{[33]}$. 在中国科学院北京基因组 研究所 Illumina HiSeq 2000平台上对实验组和对照组 进行文库测序.

\section{5 转录组组装和功能基因注释}

cDNA文库测序得到的原始序列经过包括去除低 质量reads、微生物序列污染(真菌/细菌/病毒)和修剪 接头序列的质量检测和数据再加工后使用短reads组 装软件Trinity (version 2013) 按照默认参数进行转录组 de novo 组装产生 contigs. 随后组装好的序列通过Cd-hit 聚类软件被进一步聚类和去除冗余序列. 篮除掉细菌 蛋白和真菌蛋白序列后所有 unigenes与NCBI的非圥余 蛋白数据库(http://www.ncbi.nlm.nih.gov/)和Swiss-prot 数据库进行BLASTX比对 $\left(E\right.$-value $\left.<10^{-5}\right)$. 此外, 为了确 认富集的 GO terms, 所有的 unigenes都使用BLAST2GO 对 $\mathrm{GO}$ 数据库进行了基因本体 $(\mathrm{GO})$ 富集分析. 最后, 使用KEGG (Kyoto Encyclopedia of Genes and Genomes database)构建富集通路和代谢网络 ${ }^{[34]}$. 用RSEM计算 FPKM值获得表达量数据(网络版附表 1 ) ${ }^{[35]}$. 差异表达 基因(DEGs) 由临界 $P<0.001$ 的 $\mathrm{R}$ 包差异表达基因序列 䇻选出 ${ }^{[36]}$. 如果这些基因的 $P<0.05$ 且表达量为对照组 的 2 倍以上或 $1 / 2$ 以下, 则可认为这些基因为差异表达 基因 ${ }^{[37]}$.

\section{6 主要差异表达转录物 (DETs) 的实时苂光定 量PCR分析和聚类分析}

为确认转录组测序的表达谱结果, 从中选取了 几个主要的免疫相关基因, 设计了特异性引物(网络 版附表2), 并对其进行实时荧光定量PCR 分析. 使用 FastQuant RT Kit (Tiangen, 北京)试剂盒加 $1 \mu \mathrm{g}$ 总RNA 对其进行cDNA合成. 使用SYBR green PCR Master Mix 在 MX3000P系统上进行定量实时 PCR; 热循环条件为: $95^{\circ} \mathrm{C}, 5 \mathrm{~s} ; 55^{\circ} \mathrm{C}, 30 \mathrm{~s} ; 72^{\circ} \mathrm{C}, 30 \mathrm{~s}$ (40个循环). 用R语言环 境中的 gplots工具包里的heatmap. 2 功能对 $q P C R$ 数据进 行聚类分析.

\section{2 结果与分析}

\section{1 感染不同真菌的松墨天牛的死亡率}

在注射和接触试验中感染 $S$. sp. 1 和球狍白僵菌的 松墨天牛蛹均呈现不同的反应. 结果表明, 注射 $S$. sp. 1 的蛹对真菌的免疫应答和发育情况与对照组无明显 差异. 这两种处理条件下的蛹均可继续发育成成虫. 然而, 注射球孢白僵菌的蛹在感染 $24 \mathrm{~h}$ 内伤口周围变 黑, 在感染 $48 \mathrm{~h}$ 后蛹的存活率只有 $23 \%$. 感染 $72 \mathrm{~h}$, 所有 蛹全部变黑并死亡. 1 个月后, 蛹被完全包裹在真菌菌 丝体和分身狍子中(图1A和B). 在接触试验中也可观 察到相似的结果. 5 天后, $S$. sp. 1 和球孢白僵菌菌丝体 开始入侵蛹体. 在第 10 天, 球孢白僵菌培养皿中的蛹 全部死亡, 并被完全包裹在菌丝体内. 与之相反, $S$. sp. 1 培养血中的蛹均存活并可正常发育直至羽化为成虫.

\section{2 注射不同真菌后松墨天牛转录组差异表达的 鉴定和功能分析}

为研究感染不同真菌的松墨天牛死亡率差异产 生的原因, 本实验构建了感染真菌的松墨天牛cDNA 文库, 并进行了基因差异表达分析. 根据BLASTX搜 索比对非冗余(nonredundant, NR)序列数据库, 注释了 55059 条 unigenes. 试验表明, 感染 S. sp.1的天牛与未注 射真菌的天牛(对照组)具有相似的表达模式, 表示天 牛蛹可适应共生菌 $S$. sp.1并保持正常生理状态. 此外, 鉴定出 5 个主要的基因簇, 这 5 个基因簇随时间呈现出 不同的表达模式(图2A)。感染球狍白僵菌的天牛，第 

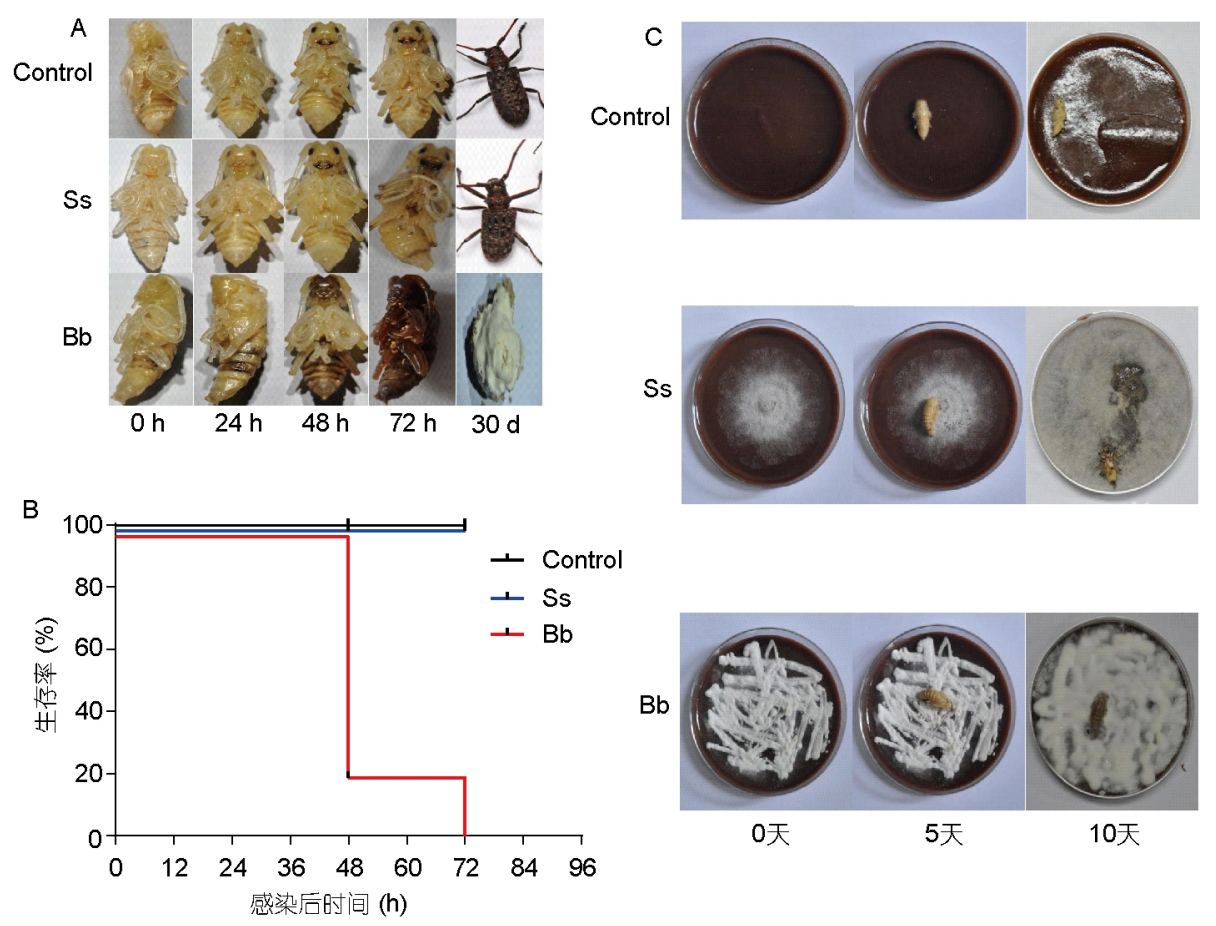

图 1 感染球狍白僵菌和 $S$. sp. 1 的松墨天牛的不同存活响应

A: 感染 $S$. sp. 1 和球孢白僵菌的天牛的不同表型. 感染 $S$. sp. 1 的天牛不会死亡且可正常发育成成虫,而感染球孢白僵菌的天牛会在注射 $24 \mathrm{~h}$ 后死亡; B: 松墨天牛感染S. sp. 1(蓝线)、球狍白僵菌(红线)与对照组(黑线)的生存曲线; C: S. sp. 1和球狍白僵菌接触试验中松墨天牛的表型

1基因簇在注射 $24 \mathrm{~h}$ 时有 19151 个unigenes上调; 第3 基 因簇在注射 $48 \mathrm{~h}$ 后 11969 个 unigenes 上调. 感染 $S$. sp. 1 的天牛, 第2基因族中的大量基因(9576个unigenes)主 要在注射 $24 \mathrm{~h}$ 后表达; 然而, 除了第 4 基因族(6702个 unigenes), 大多数基因在感染 $48 \mathrm{~h}$ 后趋于正常表达. 这 些结果表明, 刚感染 $S$. sp. 1 与注射 $S$. sp. $148 \mathrm{~h}$ 后天牛 的基因表达规律不同, 注射 $48 \mathrm{~h}$ 后基因表达规律更接 近于对照组样本.

与对照组相比, 注射球狍白僵菌 $24 \mathrm{~h}$ 后 10122 个转 录本受到显著调控(5453个上调转录本和4669个下调 转录本), 而注射 $48 \mathrm{~h}$ 后更多转录本被显著调控(8406 个上调转录本和 5309 个下调转录本). 随着感染时间 的增加, 天牛的基因表达变得更为活跃. 在球孢白僵 菌注射的 2 个时间点, 共有 5967 个共同差异表达基因 $(2605+239+298+2825)$ (图2B). S. sp. 1感染的松墨天牛 蛹, 注射 $24 \mathrm{~h}$ 后共有 5111 个转录本上调, 4179 个下调, 注射 $48 \mathrm{~h}$ 后共有 2493 个转录本上调, 4267 个下调, 发 生显著改变(图2C). 这些结果表明, 球孢白僵菌和 $S$. sp. 1感染天牛 $24 \mathrm{~h}$ 后基因表达水平均明显上调; 而两 种真菌感染 $48 \mathrm{~h}$ 后, 天牛则表现出显著的基因差异表
达: 球孢白僵菌感染 $48 \mathrm{~h}$ 时松墨天牛的基因表达调控 (5503+2605+298 个基因上调表达, 2245+239+2825个基 因下调表达)明显较 $S$. sp. 1感染 $48 \mathrm{~h}$ 的基因表达调控 $(1045+1309+139$ 个基因上调表达, $1852+281+2134$ 个基 因下调表达)更为活跃. 这些结果与转录FPKM值的聚 类热图相对应. 在 $24 \mathrm{~h}$ 时 $\mathrm{Bb}$ 和 $\mathrm{Ss}$ 组的数据聚成一类, 在 $48 \mathrm{~h}$ 时与基因表达最活跃的 $\mathrm{Bb}$ 组聚成一类; 与此同时, 在 $48 \mathrm{~h}$ 时 $\mathrm{Ss}$ 组和对照组聚成一类(图 $2 \mathrm{~A}$ ).

为进一步了解DETs 的潜在功能和所涉通路, 对 DETs进行了基因本体(GO)富集和KEGG分析. GO功 能注释有 3 个部分, 包括细胞成分、分子功能和生物 过程. 结果表明, 大部分DETs 具有分子功能(催化活 性、结合)和生物过程(代谢过程、细胞过程) (图2D). 在 $\mathrm{Ss}$ 和 $\mathrm{Bb}$ 组中上调DETs的KEGG通路结果表明, 大部 分DETs主要参与转录、折叠、分选和降解、复制和 修复、碳水化合物代谢、酶家族、信号转导、信号 分子和相互作用(图2E). GO和KEGG分析结果表明, 真 菌感染影响了昆虫的总代谢活动和相应细胞反应. 在 球孢白僵菌感染松墨天牛 $48 \mathrm{~h}$ 时其基因表达调控最为 活跃, 而 $S$. sp. 1 感染 $48 \mathrm{~h}$ 时, 松墨天牛的基因表达最不 

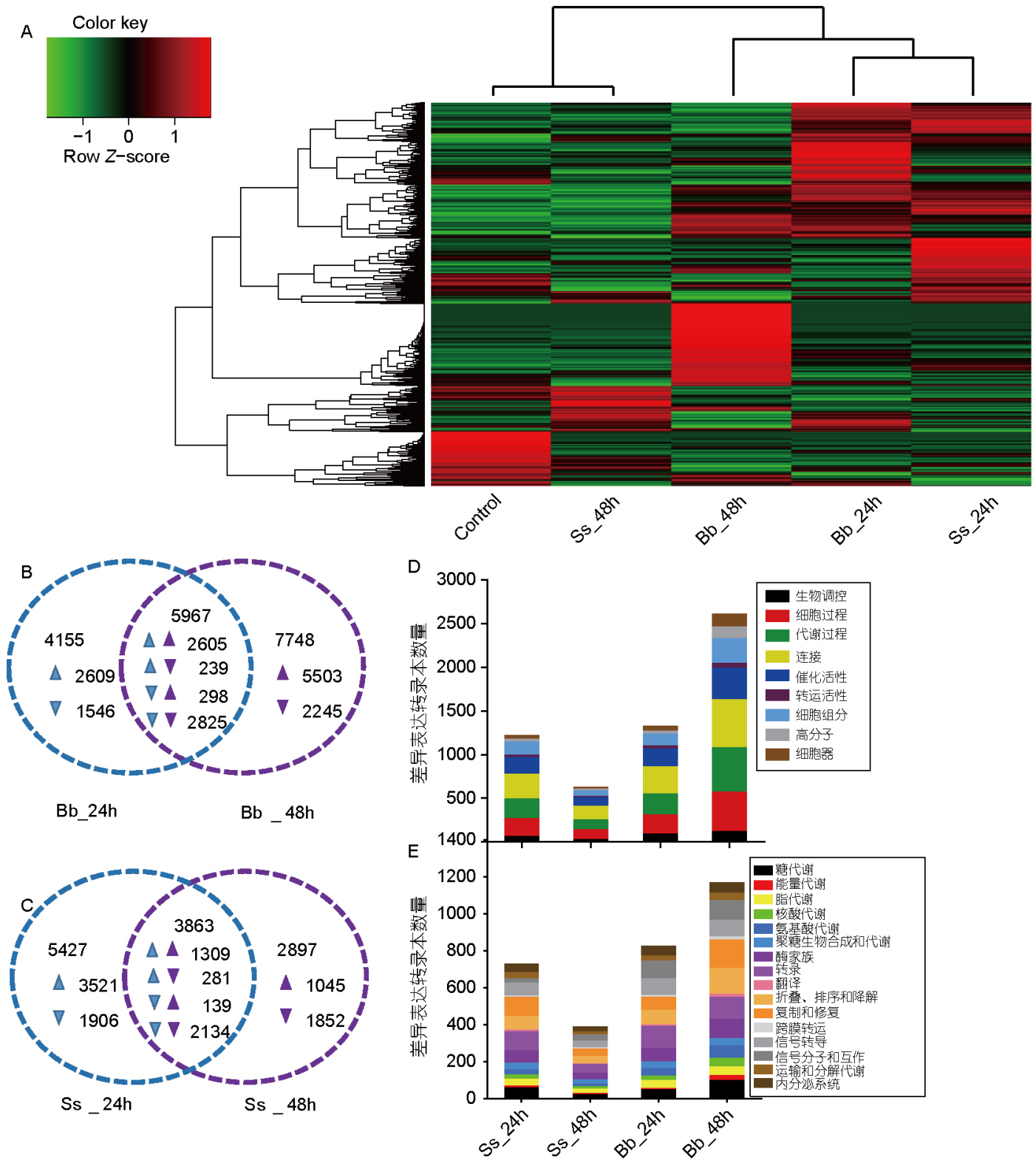

图 2 松墨天牛感染 $S$. sp. 1和球狍白僵菌的转录组差异

A: 感染 $S$. sp. 1 和球孢白僵菌 24 和 $48 \mathrm{~h}$ 的松墨天牛转录组的差异表达转录物(DETs)的聚类分析; B: 球狍白僵菌感染天牛 24 和 $48 \mathrm{~h}$ 的DETs数 据的维恩图; C: $S$. sp. 1 感染天牛 24 和 $48 \mathrm{~h}$ 的DETs数据的维恩图; D: 感染 $S$. sp. 1 和球孢白僵菌的天牛的差异表达基因 GO功能分类; E: 感染 $S$. sp. 1和球狍白僵菌的天牛差异表达上调基因的KEGG功能分类

活跃, 处于非感染阶段.

\section{3 松墨天牛免疫相关基因的分类和表达谱}

转录组测序共得到55059个unigenes, 通过BLASTX 比对, 共注释出197个松墨天牛免疫相关基因. 这些 基因包括病原体识别分子、信号调节分子、细胞内
信号转导分子、免疫应答效应分子和细胞免疫基因。 约 $32.49 \%$ 的细胞内信号转导分子组与 Toll, IMD, JNK 和JAK/STAT通路有关. 在该组中, Toll通路中鉴定出 20 个免疫相关基因, IMD通路中鉴定出 22 个免疫相关 基因, 这两个信号通路是昆虫免疫中的主要信号通路 (图3A). 

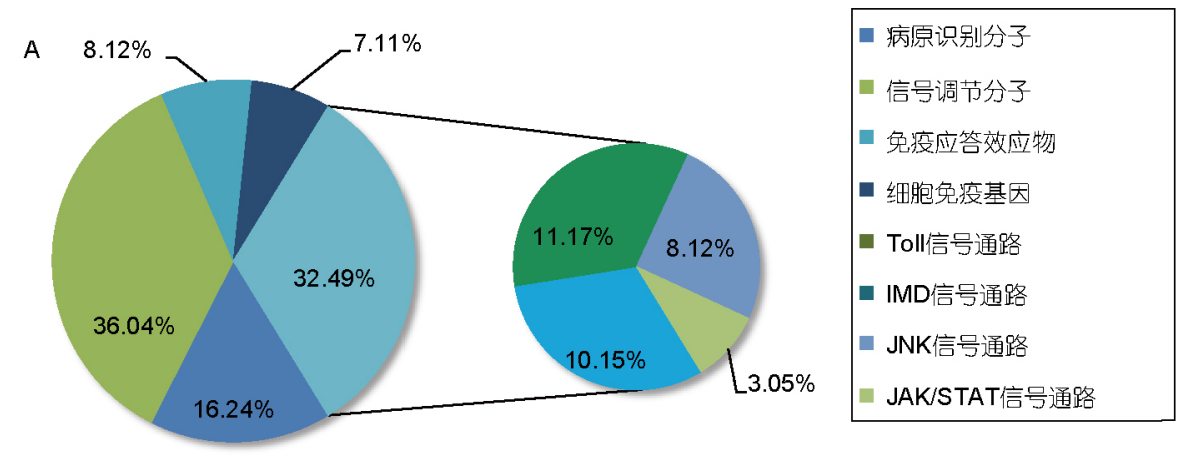

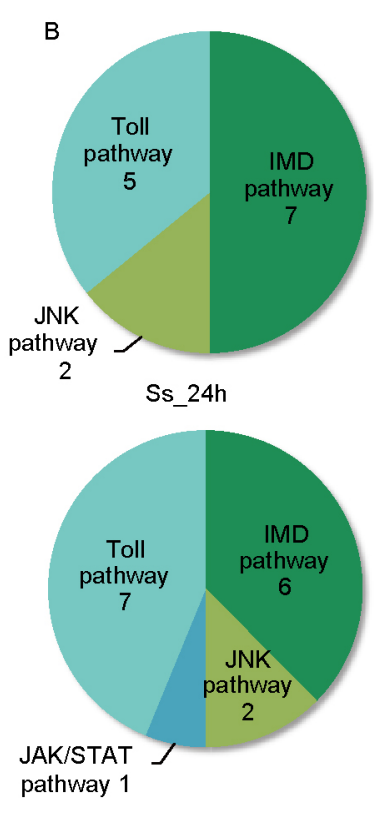

Bb_24h

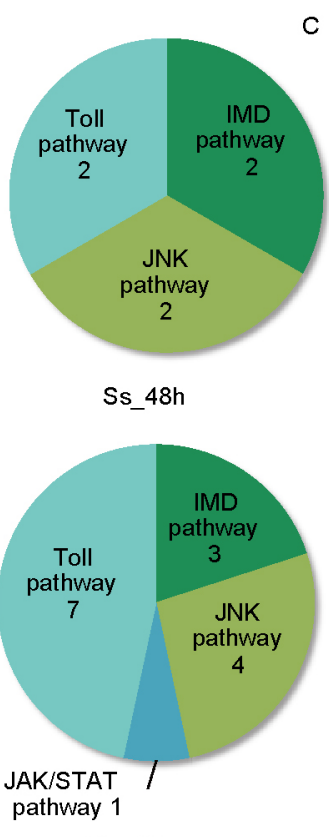

Bb_48h

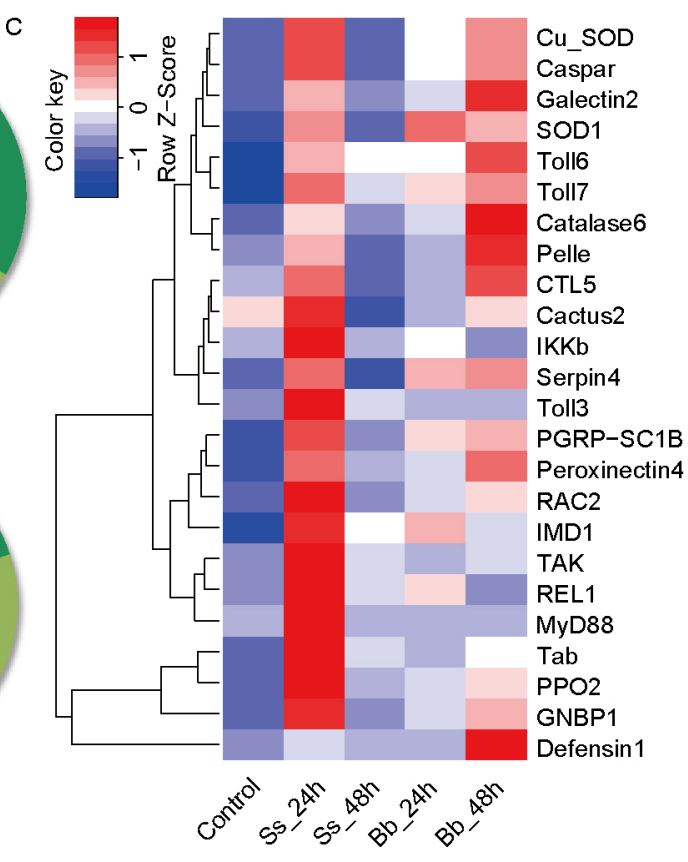

图 3 松墨天牛免疫相关基因特征描述

A: 松墨天牛免疫相关基因功能的主要类别包括: 病原识别、信号调控、胞内信号转导、免疫应答和细胞免疫; B: 松墨天牛感染 $S$. sp. 1和 球孢白僵菌 24 和 $48 \mathrm{~h}$ 后胞内信号通路中DETs分配情况; C: 松墨天牛主要差异表达基因的聚类分析, 脂肪体样本分为 3 组: $S$. sp. 1 组、 球孢白僵菌组和对照组. 蓝色、白色、红色代表mRNA相对表达量的低、中、高水平

因此, 从转录组差异表达基因中笁选出包括Toll 和IMD通路基因在内的细胞内信号转导分子进行进 一步的分析. S. sp. 1感染 $24 \mathrm{~h}$ 后, 从松墨天牛DETs中 检测到 14 个主要参与 Toll和IMD通路的基因. 经 $S$. sp. 1 感染 $48 \mathrm{~h}$ 后, DETs的数量快速下降, 尤其是参与Toll 和IMD通路的基因. 相比之下, 球狍白僵菌感染 $24 \mathrm{~h}$ 后的DETs数量较高, 有 16 个. 但 $48 \mathrm{~h}$ 后, Ss组( 6 个)的细 胞内信号转导DETs比Bb组(15个)少. 球孢白僵菌感染 $24 \mathrm{~h}$ 后天牛的细胞内信号转导DETs与感染 $48 \mathrm{~h}$ 后数量 相似, 均以Toll通路占主导地位(图3B).

\section{4 所选免疫相关基因的实时定量PCR分析}

此外, 本研究对选定的免疫相关基因进行实时定 量PCR分析, 以验证对照组和实验组间的免疫应答变 化. 结果表明, S. sp. 1感染的天牛Toll (Toll, MyD88, Pelle, Cactus)和IMD (IMD, Tab, Tak, Ikkb, Rel)信号通 路在感染 $24 \mathrm{~h}$ 后高水平表达, 但是这些免疫相关基因 的表达在感染 $48 \mathrm{~h}$ 后恢复正常水平, 表明松墨天牛对 S. sp. 1产生免疫耐受. 然而, 在球孢白僵菌感染的蛹 中, 免疫相关基因的表达水平在 $24 \mathrm{~h}$ 时变化不明显. 值 得注意的是, 免疫识别和Toll信号通路相关基因随时 
间推移明显活跃, 而 IMD途径中的基因在 $48 \mathrm{~h}$ 几乎没 有被激活(图3C).

\section{3 讨论}

共生微生物与其宿主的相互作用有利于保持宿主 的生理、代谢和免疫稳态 ${ }^{[38]}$. 宿主的先天免疫也可以 保持共生体系中免疫耐受的微妙平衡. 虽然共生关系 存在于大多数后生动物中, 但体外共生关系中寄主的 免疫机制尚不明确. 本研究发现, 松墨天牛对外共生真 菌S. sp. 1 引发的感染具有免疫耐受性. 根据感染 $S$. sp. 1不同时段的松墨天牛免疫相关基因表达谱, 天牛在感 染蓝变菌早期会引发急性免疫反应, 但随时间延长松 墨天牛通过抑制对蓝变菌的免疫反应产生免疫耐受并 保持正常发育; 松墨天牛免疫相关基因表达的热图也 与对照组非常相似. 巧合的是, 这种模式的共生关系 在肠道微生物中也非常常见, 即宿主形成一套复杂的 免疫机制以耐受共生关系中的微生物 ${ }^{[39,40]}$. PGRP-LC 抑制子(PIMS)是果蝇(Drosophila melanogaster) IMD信 号通路的一种调控子, 可减少质膜中肽聚糖识别蛋白 (PGRP-LC) 的释放, 从而阻断果蝇的IMD信号通路, 使 果蝇对共生细菌产生免疫耐受 ${ }^{[41]}$. 以前的研究发现, 在昆虫和内共生真菌互作时也产生类似的免疫耐受 机制. 小金蝠蛾(Hepialus xiaojinensis)幼虫对内共生真 菌冬虫夏草(Ophiocordyceps sinensis) 具有免疫耐受, 感 染时间越长小金蝠蛾的免疫基因表达越趋于正常水 平 ${ }^{[42]}$. 然而, 大多数有关免疫逃避的研究都着眼于内 共生系统, 本研究则更侧重宿主感染外共生真菌时产 生的免疫反应.

大多数共生真菌在感染早期不仅不会杀死宿主, 反而对宿主有一定益处 ${ }^{[10]}$. 这与昆虫病原真菌(如球 孢白僵菌)是不同的. 本研究表明, 感染 $24 \mathrm{~h}$ 时, 松墨天
牛对球狍白僵菌的免疫反应是无活性的. 而感染 $48 \mathrm{~h}$ 时, Toll信号通路的基因迅速激活, 使天牛在感染几天 内死亡. 类似地, 以前的研究表明球孢白僵菌自然感 染状态下的果蝇会选择性激活Toll通路中编码抗菌活 性物质的基因, 使其表达 ${ }^{[43]}$. 然而对共生真菌感染, 宿 主显示出不同的免疫反应: 感染蓝变菌 $S$. sp. 1 的松墨 天牛在 $24 \mathrm{~h}$ 时主要激活IMD信号通路和Toll信号通路. 在 $48 \mathrm{~h}$ 时这些免疫基因便失去活性, 使得宿主与共生 微生物能够维持二者之间的共生关系.

许多研究报道, 两种共生 ophiostomatoid蓝变菌 Ophiostoma minus 和Trichoderma sp. 可明显提升宿主 松墨天牛携带PWN的数量 ${ }^{[4]}, S$. sp. 1 也对天牛的生长 发育有积极的影响 ${ }^{\left[{ }^{5}\right.}$. 在转录组水平, 本实验发现在 $S$. sp. 1感染松墨天牛 $24 \mathrm{~h}$ 后有 9290 个转录本被激活. 大 部分DETs 具有分子功能和生物过程, 多数上调表达基 因主要表现为转录、折叠、分选和降解、复制和修 复、碳水化合物代谢、信号转导、信号分子和相互 作用等. 简单来说, 可推测 $S$. sp. 1 可能加速了松墨天 牛的新陈代谢且 $S$. sp. 1感染可能导致天牛的免疫反 应和生理新陈代谢. 松墨天牛的免疫耐受可能与 $S$. sp. 1 对天牛发育的积极影响有关, 还有待日后研究阐明.

松墨天牛与本地共生真菌S. sp. 1的互作研究是“松 材线虫-松墨天牛-蓝变菌”共生体系的重要组成部分. 但松墨天牛基因组序列的缺少是研究“松材线虫-松墨 天牛-蓝变菌”互作的分子生物学的巨大障碍. 本实验 通过转录组测序得到55059个 unigenes, 通过BLASTX 比对注释出 197 个免疫相关基因, 其中包括Toll和IMD 途径中的许多关键基因. 功能基因的鉴定和注释将为 未来研究天牛对其共生真菌免疫作用的分子机制提 供基础, 为研究松材线虫病系统提供一个新的方向, 以更好地了解林业害虫与真菌的相互作用.

\section{参考文献}

1 Alves M, Pereira A, Matos P, et al. Bacterial community associated to the pine wilt disease insect vectors Monochamus galloprovincialis and Monochamus alternatus. Sci Rep, 2016, 6: 23908

2 Vicente C, Espada M, Vieira P, et al. Pine Wilt Disease: a threat to European forestry. Eur J Plant Pathol, 2012, 133: 89-99

3 Enda N, Mamiya Y. Transmission of Bursaphelenchus Lignicolus (Nematoda: Aphelenchoididae) by Monochamus Alternatus (Coleoptera: Cerambycidae). Nematologica, 1972, 18: 159-162

4 Suh D Y, Hyun M W, Kim J J, et al. Ophiostoma ips from Pinewood Nematode Vector, Japanese Pine Sawyer Beetle (Monochamus alternatus), in Korea. Mycobiology, 2013, 41: 59-62

5 Zhao L, Zhang S, Wei W, et al. Chemical signals synchronize the life cycles of a plant-parasitic nematode and its vector beetle. Curr Biol, 2013, 
23: 2038-2043

6 Dori-Bachash M, Avrahami-Moyal L, Protasov A, et al. The occurrence and pathogenicity of Geosmithia spp. and common blue-stain fungi associated with pine bark beetles in planted forests in Israel. Eur J Plant Pathol, 2015, 143: 627-639

7 Lu M, Hulcr J, Sun J. The role of symbiotic microbes in insect invasions. Annu Rev Ecol Evol Syst, 2016, 47: 487-505

8 Repe A, Kirisits T, Piškur B, et al. Ophiostomatoid fungi associated with three spruce-infesting bark beetles in Slovenia. Ann For Sci, 2013, 70: $717-727$

9 Zhao L, Mota M, Vieira P, et al. Interspecific communication between pinewood nematode, its insect vector, and associated microbes. Trends Parasitol, 2014, 30: 299-308

10 Hartley S E, Gange A C. Impacts of plant symbiotic fungi on insect herbivores: mutualism in a multitrophic context. Annu Rev Entomol, 2009, 54: $323-342$

11 Currie C R, Wong B, Stuart A E, et al. Ancient tripartite coevolution in the attine ant-microbe symbiosis. Science, 2003, 299: 386-388

$12 \mathrm{Xu}$ L, Lou Q, Cheng C, et al. Gut-associated bacteria of Dendroctonus valens and their involvement in verbenone production. Microb Ecol, 2015, 70: $1012-1023$

13 Schmidt O, Theopold U, Strand M. Innate immunity and its evasion and suppression by hymenopteran endoparasitoids. Bioessays, 2001, 23: 344-351

14 Strand M R, Pech L L. Immunological basis for compatibility in parasitoid-host relationships. Annu Rev Entomol, 1995, 40: 31-56

15 Gillespie J P, Kanost M R, Trenczek T. Biological mediators of insect immunity. Annu Rev Entomol, 1997, 42: 611-643

16 Lowenberger C. Innate immune response of Aedes aegypti. Insect Biochem Mol Biol, 2001, 31: 219-229

17 Muta T, Iwanaga S. The role of hemolymph coagulation in innate immunity. Curr Opin Immunol, 1996, 8: 41-47

18 Baeg G H, Zhou R, Perrimon N. Genome-wide RNAi analysis of JAK/STAT signaling components in Drosophila. Genes Dev, 2005, 19: $1861-1870$

19 Valanne S, Wang J H, Rämet M. The Drosophila Toll signaling pathway. J Immunol, 2011, 186: 649-656

20 Silverman N, Maniatis T. NF-kappaB signaling pathways in mammalian and insect innate immunity. Genes Dev, 2001, 15: 2321-2342

21 Rämet M, Lanot R, Zachary D, et al. JNK signaling pathway is required for efficient wound healing in Drosophila. Dev Biol, 2002, 241: 145-156

22 Agaisse H, Petersen U M, Boutros M, et al. Signaling role of hemocytes in Drosophila JAK/STAT-dependent response to septic injury. Dev Cell, 2003, 5: 441-450

23 De Gregorio E, Spellman P T, Tzou P, et al. The Toll and Imd pathways are the major regulators of the immune response in Drosophila. EMBO J, 2002, 21: 2568-2579

24 Zou Z, Evans J D, Lu Z, et al. Comparative genomic analysis of the Tribolium immune system. Genome Biol, 2007, 8: R177

25 Lemaitre B, Nicolas E, Michaut L, et al. The dorsoventral regulatory gene cassette spätzle/Toll/cactus controls the potent antifungal response in Drosophila adults. Cell, 1996, 86: 973-983

26 Lemaitre B. Landmark: the road to Toll. Nat Rev Immunol, 2004, 4: 521-527

27 Garver L S, de Almeida Oliveira G, Barillas-Mury C. The JNK pathway is a key mediator of Anopheles gambiae antiplasmodial immunity. PLoS Pathog, 2013, 9: e1003622

28 Park J M, Brady H, Ruocco M G, et al. Targeting of TAK1 by the NF-kappaB protein Relish regulates the JNK-mediated immune response in Drosophila. Genes Dev, 2004, 18: 584-594

29 Ferron P. Biological control of insect pests by entomogenous fungi. Annu Rev Entomol, 1978, 23: 409-442

30 Wraight S P, Carruthers R I, Jaronski S T, et al. Evaluation of the entomopathogenic fungi Beauveria bassiana and Paecilomyces fumosoroseus for microbial control of the silverleaf whitefly, Bemisia argentifolii. Biol Control, 2000, 17: 203-217

31 Gottar M, Gobert V, Matskevich A A, et al. Dual detection of fungal infections in Drosophila via recognition of glucans and sensing of virulence factors. Cell, 2006, 127: 1425-1437

32 Yassine H, Kamareddine L, Osta M A. The mosquito melanization response is implicated in defense against the entomopathogenic fungus Beauveria bassiana. PLoS Pathog, 2012, 8: e1003029

33 Wang Y H, Hu Y, Xing L S, et al. A critical role for CLSP2 in the modulation of antifungal immune response in mosquitoes. PLoS Pathog, 2015, 11: e1004931

34 Hou Y, Wang X L, Saha T T, et al. Temporal coordination of carbohydrate metabolism during mosquito reproduction. PLoS Genet, 2015, 11: e1005309

35 Tu Q, Cameron R A, Worley K C, et al. Gene structure in the sea urchin Strongylocentrotus purpuratus based on transcriptome analysis. Genome Res, 2012, 22: 2079-2087 
Wang L, Feng Z, Wang X, et al. DEGseq: an R package for identifying differentially expressed genes from RNA-seq data. Bioinformatics, 2010, 26: $136-138$

37 Xiong G H, Xing L S, Lin Z, et al. High throughput profiling of the cotton bollworm Helicoverpa armigera immunotranscriptome during the fungal and bacterial infections. BMC Genomics, 2015, 16: 321

38 Bäckhed F, Ley R E, Sonnenburg J L, et al. Host-bacterial mutualism in the human intestine. Science, 2005, 307: 1915-1920

39 Müller C, Autenrieth I, Peschel A. Intestinal epithelial barrier and mucosal immunity. Cell Mol Life Sci, 2005, 62: 1297-1307

40 Macdonald T T, Monteleone G. Immunity, inflammation, and allergy in the gut. Science, 2005, 307: 1920-1925

41 Lhocine N, Ribeiro P S, Buchon N, et al. PIMS modulates immune tolerance by negatively regulating Drosophila innate immune signaling. Cell Host Microbe, 2008, 4: 147-158

42 Meng Q, Yu H Y, Zhang H, et al. Transcriptomic insight into the immune defenses in the ghost moth, Hepialus xiaojinensis, during an Ophiocordyceps sinensis fungal infection. Insect Biochem Mol Biol, 2015, 64: 1-15

43 Lemaitre B, Reichhart J M, Hoffmann J A. Drosophila host defense: differential induction of antimicrobial peptide genes after infection by various classes of microorganisms. Proc Natl Acad Sci USA, 1997, 94: 14614-14619

44 Maehara N, Futai K. Population changes of the pinewood nematode, Bursaphelenchus xylophilus (Nematoda: Aphelenchoididae), on fungi growing in pine-branch segments. Appl Entomol Zool, 2000, 35: 413-417 